\title{
Adjustable Load-Modulation Asymmetric Doherty Amplifier Design Using Nonlinear Embedding
}

\author{
Haedong Jang, Patrick Roblin, and Christophe Quindroit \\ The Ohio State University, Columbus, $\mathrm{OH}$
}

\begin{abstract}
We developed a new asymmetric Doherty load modulation matching network using identical transistors for the main and auxiliary amplifiers. Asymmetric Doherty power amplifiers (PA) require a larger size transistor for the auxiliary PA than for the main PA to provide the higher power and wider load modulation range. Additional impedance transformers are introduced to alleviate this requirement when using identical devices. The drain bias voltage of the main amplifier is also reduced to achieve a wider back-off. Furthermore, a large-signal model-based nonlinear embedding method is applied to predict the input and output harmonic terminations, removing the need for the multi-harmonic source/load pull characterization. An asymmetric Doherty amplifier was built using two $15 \mathrm{~W}$ peak power packaged GaN transistors of the same size. $71 \%$ drain efficiency at the peak power of $41.8 \mathrm{dBm}$ and $62.7 \%$ at the second peak of $32.8 \mathrm{dBm}$ (9 dB back-off) were observed. Above $50 \%$ drain efficiency was maintained over an $11 \mathrm{~dB}$ power range. $51.86 \%$ average drain efficiency was observed after linearization

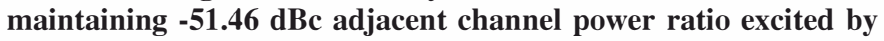
$10 \mathrm{MHz}$ bandwidth long term evolution signals with $9.96 \mathrm{~dB}$ peak to average power ratio.
\end{abstract}

Index Terms-Asymmetric, Doherty, embedding, load modulation, nonlinear, power amplifiers.

\section{INTRODUCTION}

High data rate communication environment requires spectrally efficient modulation schemes with a high peak to average power ratio (PAPR). Typical RF power amplifiers have peak efficiency only at peak power and the efficiency is reduced at backed off power levels resulting in a degraded average efficiency. Doherty amplifiers have attracted RF power amplifier designers due to their improved average efficiency with a relatively simple structure [1]. However, the conventional Doherty amplifier provides only $6 \mathrm{~dB}$ back-off level while the signals such as long term evolution (LTE) or multiple carrier wide-band code division multiple access (WCDMA) exhibit peak to average power ratio (PAPR) over $10 \mathrm{~dB}$. Asymmetric Doherty amplifiers have been demonstrated to extend the back-off range while yielding high average efficiency [1][2][3]. However asymmetric Doherty amplifiers require a larger transistor device for the auxiliary amplifiers than for the main amplifier to provide the higher power and wider load modulation range [1][2]. Even for the conventional $6 \mathrm{~dB}$ Doherty amplifiers a larger auxiliary device is preferable to compensate for the lost gain due to reduced conduction angle of class-C bias.

In this work, an additional impedance transformation is introduced to extend the load modulation range, alleviating the requirement for a large auxiliary device. Also a reduced drain voltage for the main amplifier is used to saturate it earlier. A

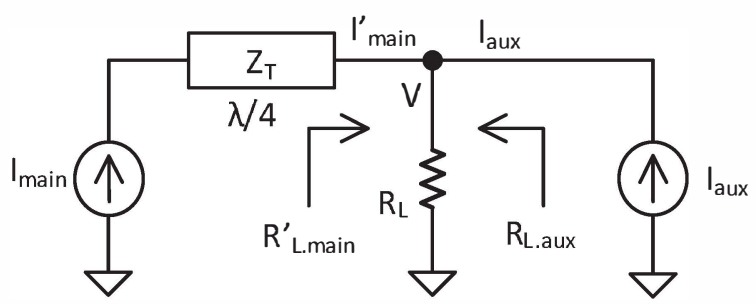

Fig. 1. The effective loads seen by the main and auxiliary amplifiers are varied by current contributions from each amplifier.

high $9 \mathrm{~dB}$ back-off asymmetric Doherty performance can then be achieved using identical devices.

The input/output harmonic terminations and input fundamental matching have critical effects for the overall amplifier performances [2] requiring intensive source/load pull characterization. This is even more essential when the main amplifier is designed to operate in class-F. Raffo et. al. introduced a PA design technique at the transistor intrinsic current source reference planes to predict the optimal harmonic terminations at the transistor external reference planes while removing the need to perform time-consuming iterative multi-dimensional harmonic source/load pull either in simulation or experimentally [4][5]. To the best of authors' knowledge, this direct PA design method is applied to the first-pass design of an asymmetric Doherty amplifier for the first time.

The additional impedance transformation and the effects of the reduced drain voltage are introduced in section II. The design procedure and the nonlinear embedding are detailed in section III. The measured results are discussed in section IV, followed by conclusions in section V.

\section{Adjustable load Modulation}

\section{A. A New Load Modulation Matching Network}

The main and auxiliary amplifiers are modeled as current sources in Fig. 1. The effective load seen by each amplifier is varied by the current contribution of the other amplifier. If we assume that (1) the auxiliary amplifier provides $n$ times more current than the main amplifier at the peak power and (2) their output are in-phase, the effective loads seen from each amplifier can be calculated as:

$$
R_{\text {L.main }}^{\prime}=(n+1) R_{L} \text { and } R_{L . a u x}=\frac{(n+1)}{n} R_{L} .
$$

In the conventional Doherty design, the main amplifier experiences $2 R_{L}$ load at the peak power where $n=1$. However, if we increase $n$ for the wider back-off of asymmetric 
Doherty operation, the load seen by the auxiliary amplifier approaches the load resistance, $R_{\text {L.aux }} \approx R_{L}$, while the load seen by the main side becomes $n+1$ times larger than $R_{L}$. Since the load variation experienced by the main amplifier is $R_{\text {L.main }}=Z_{T}^{2} / R_{L \text {.main }}^{\prime}$, if we choose the characteristic impedance of the quarter wave transformer, $Z_{T}$, as the peak power load, $(n+1) R_{L}$, the load seen by the main varies from $(n+1) R_{L}$ to $(n+1)^{2} R_{L}$ [2]. Therefore, if we use the same size devices for both the main and auxiliary amplifiers, the load variation range experienced by these transistors is from $(n+1) R_{L} / n$ to $(n+1)^{2} R_{L}$ as shown in Fig. 2 (a) while for the conventional Doherty amplifiers the load variation is only from $R_{L}$ to $2 R_{L}$. For example, for the conventional Doherty with $R_{L}=25 \Omega, Z_{T}=50 \Omega$, the load variation experienced by the transistor is from $50 \Omega$ to $100 \Omega$. However, for the asymmetric Doherty with $n=2, R_{L}=25 \Omega$ and $Z_{T}=75 \Omega$ for $9.54 \mathrm{~dB}$ back off, the load variation is from $37.5 \Omega$ to $225 \Omega$.

Unlike for the ideal load modulation case where the efficiency is kept constant with increasing load, a degraded efficiency is observed when using practical devices due to their on-resistance [5]. Also, the smaller $R_{L}$ requires high gate voltage swing which may cause unwanted forward gate conduction of the high-electron-mobility transistors Schottky barrier. Therefore, the asymmetric Doherty amplifier design using same size devices is normally limited in terms of load variation range, and this constrains in turn the achievable backoff range.

To alleviate this design limitation, an additional impedance transform is introduced as shown in Fig. 2 (b). It transforms the wide range of the load variation into a reduced range more favorable for the devices using the scaling factor of $\alpha$ or $\beta$ shown in that figure. As one of the possible solutions, a quarter wave length transmission line provides a convenient way of transforming the loads into the desired range. On the other hand, it introduces a phase delay, $e^{-j k \pi}$, where $k$ is the number of the quarter wave transformers engaged. In the case of odd $k$ number, the impedance variation of the Doherty quarter wave transformer is reversed. So, an even number of section must be used in the multiple-section transformers to maintain the Doherty operation. Furthermore, by proper selection of the characteristic impedances of the transformer, the pass-band transfer characteristic can be shaped as desired [6]. In this work, a two section binomial transformer was used for a maximally flat response.

\section{B. Peak Power Rescaling with Reduced Drain Voltage}

The reduced peak power in the main amplifier required for the asymmetric Doherty design, can be obtained by reducing the drain bias voltage of the main PA. This in turns reduces the optimal resistive load for the transistor in the main PA. Assuming ideal class-B condition, the adjusting factor $\gamma$ can be defined as:

$$
\gamma=\frac{V_{d s 1 . r e d u c e d}-V_{o n}}{V_{d s 1 . n o r m a l}-V_{o n}}
$$

where, $V_{o n}$ represents the drain saturation voltage of the device. Assuming high and low loads, $R_{\text {L.high }}, R_{\text {L.low }}$, for

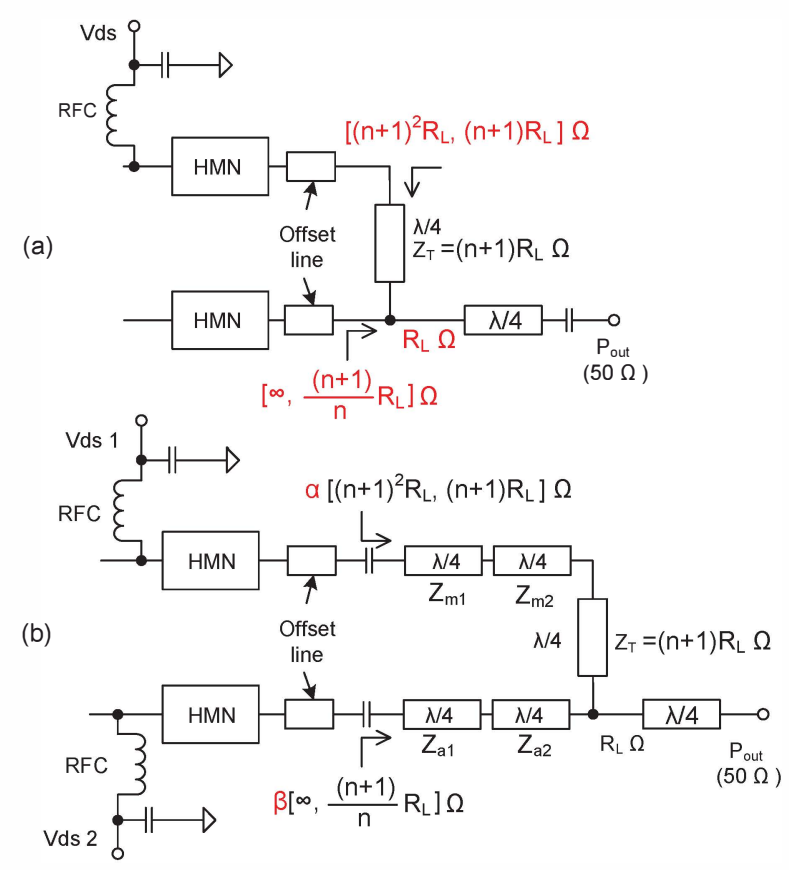

Fig. 2. Conventional asymmetric Doherty amplifier schematic (a) modified in (b) to rescale the Doherty load modulation using multiple section impedance transformers. The main amplifier drain bias voltage is reduced for early saturation. The impedances at the fundamental seen by the main and auxiliary PAs at low (left) and high (right) input powers are also indicated. Harmonics are terminated using harmonic matching networks (HMN).

the backed-off and peak power operations, respectively, the adjusted load variation range of the main amplifier is from $\gamma R_{L . l o w}$ to $\gamma R_{L \text {.high }}$. Once the favored load variation range is selected, the Doherty load, $R_{L}$ can be decided considering three design conditions for a back-off factor of $n:(1,2)$ the adjusted main load modulation needs to fall into the favorable load variation range of the main transistor and (3) the adjusted auxiliary peak power load needs to be larger than $R_{L . l o w}$.

$$
\begin{aligned}
& \gamma R_{\text {L.low }} \leq \alpha(n+1) R_{L} \\
& \gamma R_{\text {L.high }} \geq \alpha(n+1)^{2} R_{L} \\
& R_{\text {L.low }} \leq \beta\left(\frac{n+1}{n}\right) R_{L} .
\end{aligned}
$$

However, using identical devices for both main and auxiliary amplifiers, the Doherty load, $R_{L}$ in the range:

$$
\frac{n R_{L . l o w}}{\beta(n+1)} \leq R_{L} \leq \frac{\gamma R_{L . h i g h}}{\alpha(n+1)^{2}}
$$

may not exist without introducing the impedance transform ratios $\alpha$ and $\beta$. Therefore, one must use two different multiple section (even number) transformers providing the impedance transform ratios $\alpha$ and $\beta$ for the main and peaking amplifiers respectively [6].

\section{INTRINSIC DESIGN AND NONLINEAR EMBEDDING}

The large signal model used for the $15 \mathrm{~W}$ peak power $\mathrm{GaN}$ (CGH27015F, CREE Inc.) transistor was extracted to permit 
the direct PA design process [5]. The intrinsic memoryless IV model was used for the internal design and then the external harmonic terminations were obtained by embedding the linear and nonlinear parasitics [4][5]. The intrinsic main amplifier was designed for a saturated class-F operation at $2 \mathrm{GHz}$ with a class- $\mathrm{AB}$ bias condition. The drain voltage of the main amplifier was reduced to $14 \mathrm{~V}$ from the typical $28 \mathrm{~V}$ of the device to be saturated at $9.54 \mathrm{~dB}$ backed off from the peak Doherty power. The gate was biased at $-2.9 \mathrm{~V}$, resulting in 89 $\mathrm{mA}$ intrinsic quiescent current. The auxiliary amplifier was biased at $-3.9 \mathrm{~V}, 27.5 \mathrm{~V}$ for class-C operation.

An intrinsic Doherty load of $R_{L}=12.27 \Omega$ was chosen with a quarter wave length transformer of $Z_{T}=3 R_{L}=36.81 \Omega$ characteristic impedance. If we use the same transistor for both amplifiers, the transistors experience from $18.4 \Omega$ to $110.4 \Omega$ for the peak power of the auxiliary amplifier and back-off power of the main amplifier, respectively. Therefore, in this design, the main amplifier load variation was reduced by a factor $2(\alpha=0.5)$ to avoid the efficiency degradation at large loads. Thus the main amplifier experiences $55.2 \Omega$ at the backed-off power. The auxiliary load variation was increased by 1.5 times $(\beta=1.5)$ to $27.6 \Omega$ for the optimal load at the peak power.

The designed intrinsic operation was projected to the external package reference plane through the nonlinear embedding process. Thus without performing any harmonic source/load pull, the optimal second and third harmonic loads for the class-F operation at the package reference plane were synthesized. This yielded for example, $\Gamma_{L}(2 \omega)=e^{-j 2.25}$ and $\Gamma_{L}(3 \omega)=e^{-j 2.68}$ for the main device after the amplitudes of the projected harmonic loads were normalized to the unity. Those loads were rotated to a short position with a cascaded transmission line and then terminated with quarter-wave length open stubs. The input harmonic terminations were found using the same method. The procedure was repeated for the auxiliary amplifier. The two amplifier inputs were also matched at the fundamental frequency using the complex conjugate of the device input impedance at peak power.

An offset line, $19 \Omega$ with $154^{\circ}$, was designed to rotate the fundamental loads at the package reference plane for it to lie on the real line before the load-side quarter wave transformer. The same offset line was used for the auxiliary amplifier but it was fine tuned in the final design stage. Finally the embedding design model was replaced with the manufacturer (CREE Inc.) model and the design was tuned in electro-magnetic simulation to work with the asymmetric input power division $\left(\left|S_{21}\right|=\right.$ $0.652,\left|S_{31}\right|=0.749$, with 2 and 3 the inputs of the main and auxiliary amplifiers respectively). The mode projection and model switch resulted for the main amplifier in slightly modified drain voltage and quiescent current of $14.3 \mathrm{~V}$ and 74 $\mathrm{mA}$ respectively. The auxiliary amplifier was biased at $-3.6 \mathrm{~V}$, $27.8 \mathrm{~V}$ for the gate and drain voltages. The amplifier was built on 31 mil thick RT/duroid 5880 (see Fig. 3).

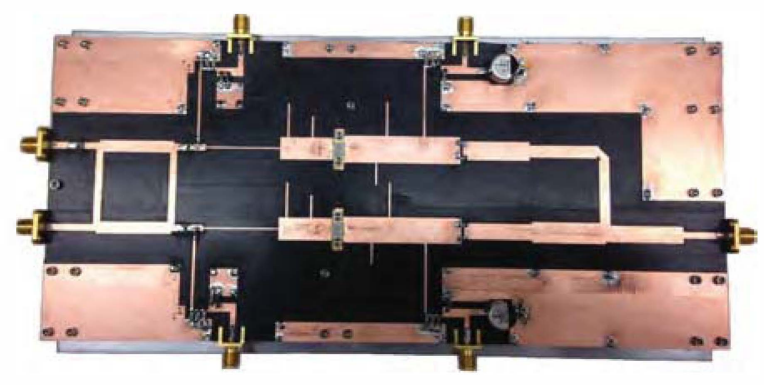

Fig. 3. The $9.54 \mathrm{~dB}$ back-off asymmetric Doherty power amplifier was built using the same two GaN transistors. The board size is $10 \times 4.5 \mathrm{in}^{2}$.

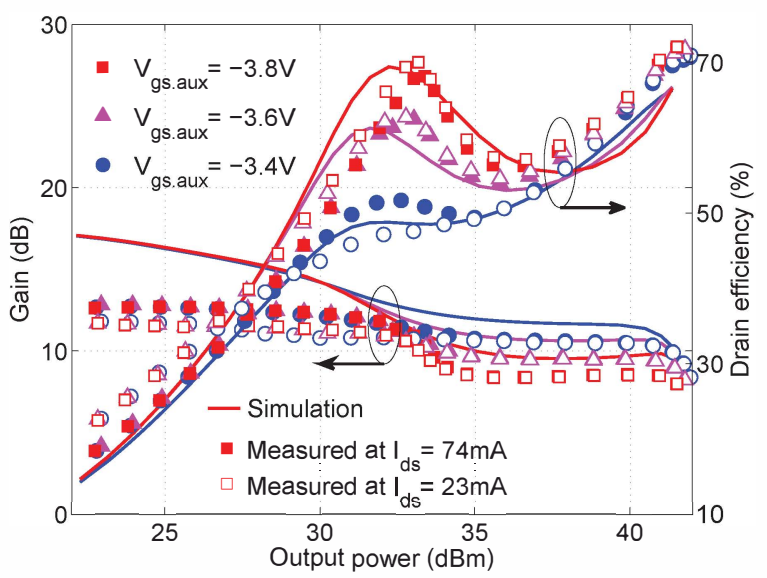

Fig. 4. Measured (solid and blank symbols) drain efficiency and gain at 2 $\mathrm{GHz}$ are compared to simulation (solid lines) with swept input powers at three different auxiliary amplifier gate voltages. Reduced main amplifier bias current from $74 \mathrm{~mA}$ (solid symbols) to $23 \mathrm{~mA}$ (blank symbols) shifts the efficiency curves to the left.

\section{MEAsured RESUlts AND Discussion}

The output power and drain currents for the main and auxiliary amplifiers were measured with swept input powers at $2 \mathrm{GHz}$ using continuous waves. Three different auxiliary amplifier gate voltages $\left(V_{g s 2}=-3.4 \mathrm{~V},-3.6 \mathrm{~V}\right.$ and $\left.-3.8 \mathrm{~V}\right)$ were varied to investigate the trade off between efficiency and linearity. A signal generator (Agilent E4438C) and a large signal network analyzer (LSNA, MT4463A) were used for the measurements. The currents were measured using an oscilloscope (Tektronix, DPO7104) with two current probes (TCP0030) for the main and auxiliary amplifiers. The probes were wound 3 times to increase current measurement sensitivity and the measured values were divided by the same number.

Fig. 4 compares the measured drain efficiency and power gain (symbols) to the simulated data (solid lines). It was observed that increasing the auxiliary gate voltage reduces the second peak efficiency while flattening the gain curves. It is interesting to note that reducing the main amplifier quiescent current to $23 \mathrm{~mA}$ from $74 \mathrm{~mA}$ slightly shifts the efficiency curves to the left resulting in wider back-off and flatter gain curves. So, the most flat gain was obtained at $-3.4 \mathrm{~V}$ for the auxiliary gate voltage and $23 \mathrm{~mA}$ for the main amplifier 


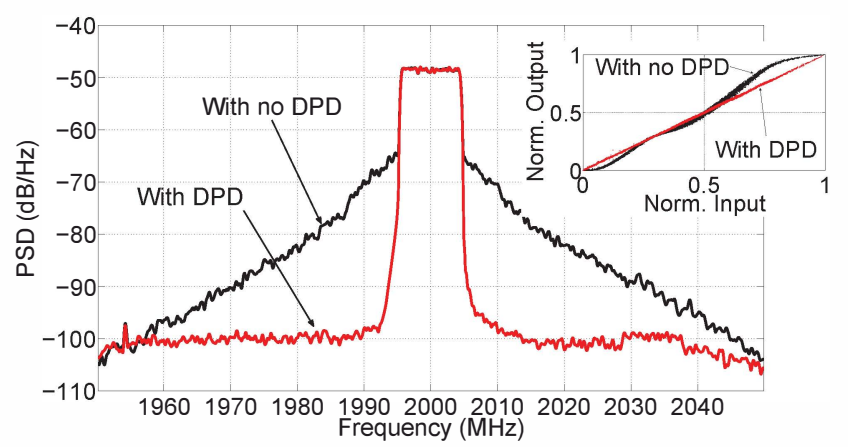

Fig. 5. Spectrum and AM-AM distortion were measured at $-3.6 \mathrm{~V}$ for the auxiliary gate voltage and $23 \mathrm{~mA}$ main amplifier bias current.

bias current condition with reduced efficiency. It should be mentioned that the $\mathrm{CW}$ gain in Fig. 4 was calculated using incident power instead of input power to be consistent with the linearization measurement system used for modulated signal excitations.

Above $50 \%$ drain efficiency was maintained over $11.4 \mathrm{~dB}$ output power range at $-3.6 \mathrm{~V}$ for the auxiliary gate voltage and $23 \mathrm{~mA}$ main amplifier bias current depicted by magenta blank triangles in the figure. $71 \%$ drain efficiency at the peak power of $41.8 \mathrm{dBm}$ and $62.7 \%$ at the second peak of $32.8 \mathrm{dBm}(9$ $\mathrm{dB}$ back-off) were observed. For these operating conditions, digital predistortion (DPD) from [7] using an FPGA test-bed in [8] was applied for demonstration purpose. A $10 \mathrm{MHz}$ bandwidth LTE signal with $9.96 \mathrm{~dB}$ PAPR was used. The same average output power of $33.55 \mathrm{dBm}$ was used before and after applying DPD for a fair comparison. 51.86\% average efficiency was observed after linearization maintaining -51.47 $\mathrm{dBc}$ adjacent channel power ratio (ACPR) and $-37.37 \mathrm{~dB}$ normalized mean square error (NMSE). The nonlinearity order was 15 and memory depth 7. The measured linearization results are summarized in table I and illustrated in Fig. 5.

TABLE I

AVERAGE EFFICIENCY BEFORE AND AFTER LINEARIZATION.

\begin{tabular}{c|c|c|c|c}
\hline & $\begin{array}{c}\mathrm{P}_{\text {o.avg }} \\
(\mathrm{dBm})\end{array}$ & $\begin{array}{c}\mathrm{DE}_{\text {avg }} \\
(\%)\end{array}$ & $\begin{array}{c}\text { ACPR } \\
(\mathrm{dBc})\end{array}$ & $\begin{array}{c}\text { NMSE } \\
(\mathrm{dB})\end{array}$ \\
\hline \hline Before DPD & 33.55 & 54.28 & -28.42 & -13.11 \\
\hline After DPD & 33.55 & 51.86 & -51.47 & -37.37 \\
\hline
\end{tabular}

\section{CONCLUSiON}

A technique for the selection of the optimal load modulation impedances in wide back-off asymmetric Doherty amplifiers using identical devices was introduced and demonstrated by building a $9.54 \mathrm{~dB}$ backed-off amplifier. Measured results showed that (1) the drain efficiency was maintained above $50 \%$ over an $11 \mathrm{~dB}$ output power range using the proposed method and that (2) an average drain efficiency of $51.86 \%$ was obtained with $-51.46 \mathrm{dBc}$ ACPR after linearization using $10 \mathrm{MHz}$ band-width LTE signals with $9.96 \mathrm{~dB}$ PAPR.

The optimal harmonic sources and loads were found by using nonlinear embedding through a single simulation resulting in a first-pass design for the Doherty power amplifier. This nonlinear embedding significantly simplified the design process and reduced the design time by removing the conventional source/load-pull process.

Given the fixed power-divider ratio used at the input, the Doherty PA could be tuned by adjusting the gate voltage of the auxiliary amplifier, which is equivalent to controlling the duty cycle of the class-C bias amplifier. This revealed a trade-off relationship between the average drain efficiency and the PA linearity. For future work, the use of a digitally controlled dualinput Doherty operation replacing the fixed power-divider as in Ref. [9] is suggested to simultaneously improve the average efficiency and the linearity.

\section{ACKNOWLEDGMENT}

This work was supported by the National Science Foundation (NSF) Collaborative Grant ECS-1129013. The authors would like to thank CREE, Inc., Durham, NC, for providing the GaN HEMT power transistor models and clamps used in this work. The authors would like to thank Y. Lin, R. D. Pond with The Ohio State University, Columbus, $\mathrm{OH}$ for their support during circuit prototyping and measurements.

\section{REFERENCES}

[1] J. Xia, X. Zhu, L. Zhang, J. Zhai, and Y. Sun, "High-efficiency GaN Doherty Power Amplifier for 100-MHz LTE-Advanced Application Based on Modified Load Modulation Network," emph IEEE Trans. Microwave Theory and Tech., vol. 61, no. 8, Aug. 2013.

[2] M. Iwamoto, A. Williams, P. Chen, A. G. Metzger, L. E. Larson, and P. M. Asbeck, "An extended Doherty amplifier with high efficiency over a wide power range," IEEE Microwave Theory Tech., vol. 49, pp. 24722479, Dec. 2001.

[3] J. Son, I. Kim, J. Moon, J. Lee, and B. Kim, "A highly efficient asymmetric Doherty power amplifier with a new output combining circuit," IEEE Int. Mircrowaves, Communications, Antennas and Electronics Systems Conference, Tel Aviv, Nov. 2011.

[4] A. Raffo, F. Scappavia, and G. Vannini, "A new approach to microwave power amplifier design based on the experimental characterization of the intrinsic electron-device load line," IEEE Microwave Theory Tech., vol. 57, no. 7, July 2009.

[5] H. Jang, P. Roblin, and Z. Xie, "Model-based direct RF power amplifiers design using intrinsic to extrinsic mode projection," (to be reported elsewhere).

[6] David M. Pozar, Microwave Engineering, 3rd ed. Hoboken, NJ: Wiley, John \& Son Inc., 2005.

[7] R. Raich, H. Qian, and G. T. Zhou, "Orthogonal polynomials for power amplifier modeling and predistorter design," IEEE Trans. on Vehicular technology, vol. 53, No. 5, Sept. 2004.

[8] C. Quindroit, N. Naraharisetti, P. Roblin, S. Gheitanchi, V. Mauer, M. Fitton, "FPGA Implementation of Orthogonal 2D Digital Predistortion System for Concurrent Dual-Band Power Amplifiers Based on TimeDivision Multiplexing," IEEE Microwave Theory and Tech. IEEE Transactions on, vol. 61, no. 12, pp. 4591-4599, Dec. 2013

[9] R. Darraji, F. M. Ghannouchi and O. Hammi, "A dual-input digitally driven Doherty amplifier architecture for performance enhancement of Doherty transmitters," Microwave Theory and Techniques, IEEE Transactions on , vol.59, no.5, pp.1284-1293, May 2011 